\title{
LA PROYECCIÓN DE LA INICIATIVA CINTURÓN Y RUTA DE LA SEDA EN LA UNIÓN EUROPEA (2014- 2019): IMPLICACIONES ECONÓMICAS Y NORMATIVAS $^{1}$
}

\author{
THE PROJECTION OF THE BELT AND ROAD INITIATIVE \\ INTO THE EUROPEAN UNION (2014-2019): ECONOMIC \\ AND NORMATIVE IMPLICATIONS
}

\section{Manuel de Jesús Rocha Pino ${ }^{2}$ \\ ORCID: 0000-0002-7295-9056}

\section{RESUMEN}

La investigación analiza las características que poseen los proyectos de la Iniciativa Cinturón y Ruta de la Seda (CyR), instrumentados durante el periodo 2014-2019 en la Unión Europea (UE), y su relevancia para el desarrollo de las relaciones políticas y económicas sino-europeas. Este proceso se ha desarrollado en un contexto de interdependencia económica creciente entre los actores, algo evidenciado por los altos volúmenes en sus intercambios comerciales. La investigación utiliza un enfoque basado en la teoría liberal-institucionalista de relaciones internacionales con la finalidad de analizar las preferencias elegidas por China y la UE para orientar su relación de una manera pragmática, aunque no exenta de asimetrías; así como los instrumentos institucionales utilizados para coordinar la agenda bilateral: la plataforma institucional que tiene la función coordinadora de las actividades del CyR en la UE es la asociación estratégica China-UE. Algunas de las conclusiones identificadas en la investigación son: 1) la UE es considerada por el gobierno chino como una región fundamental para la instrumentación del CyR, 2) la inversión en proyectos de infraestructura financiados por los capitales de origen chino tiene el objetivo de profundizar la interdependencia económica con la UE, y 3) el gobierno chino debe tener en cuenta los intereses económicos de la parte europea y el respeto a las normativas de la UE.

Palabras clave: Iniciativa Cinturón y Ruta; asociación estratégica China-Unión Europea; cooperación económica; inversión extranjera; asimetrías.

\section{ABSTRACT}

This research analyzes the characteristics of the projects of the Belt and Road Initiative (BRI) implemented during the period 2014-2019 in the European Union (EU), and their relevance for the development of Sino-European political and economic relations. This process has been developed in a context of increasing economic interdependence between the actors, something evidenced by the high volumes reached in their commercial exchanges. The research uses an approach based on the liberal-institutionalism theory in order to analyze the preferences chosen by China and the EU to guide their relationship in a pragmatic way, although not without asymmetries, as well as the institutional instruments used

1 La investigación fue elaborada durante una estancia de investigación posdoctoral en El Colegio de México con una beca otorgada por el Consejo Nacional de Ciencia y Tecnología (Conacyt). El autor agradece la asesoría de la Dra. Marisela Connelly; todos los errores y omisiones corresponden al autor.

2 El Colegio de México, Centro de Estudios de Asia y África, México. Doctor en Relaciones Internacionales e Integración Europea por la Universidad Autónoma de Barcelona. Correo electrónico: manuel.rocha@colmex.mx 
to coordinate the bilateral agenda: the institutional platform having the coordinating function of the BRI activities in the EU is the China-EU strategic partnership. Some of the conclusions identified in the research are: 1) the EU is considered by the Chinese government as a fundamental region for the implementation of the BRI; 2) the investing in infrastructure projects financed by Chinese capital has the objective of deepening economic interdependence with EU members, and 3) the Chinese government must take into account the economic interests of the European part and respect for EU regulations.

Keywords: Belt and Road Initiative; ChinaEuropean Union strategic partnership; economic cooperation; foreign investment; asymmetries.

\section{Introducción}

En este trabajo se analiza la proyección de la Iniciativa Cinturón y Ruta de la Seda (CyR - yidai-yilu, 一带一路) en el espacio geoeconómico de la Unión Europea (UE) durante el periodo 2014-2019. Igualmente se describen las características que poseen los proyectos del CyR instrumentados en el espacio europeo y su relevancia para el desarrollo de las relaciones políticas y económicas que mantienen China y la UE: la plataforma institucional coordinadora de la agenda de las actividades de inversión del CyR en la UE es la asociación estratégica China-UE, establecida a partir de 2003. Esta asociación se considera el espacio institucional en el que ambos actores han dialogado y acomodado sus intereses en temas como sus relaciones de cooperación, sus asimetrías estructurales y sus relaciones de interdependencia económica en las que sobresalen los intercambios comerciales (la principal actividad de la agenda de la asociación es la Cumbre China-UE de alto nivel) (Rocha Pino, 2018).

En el proceso de estructuración de la agenda de la asociación, para China y la UE resultan prioritarios tanto el orden (las reglas, instituciones y figuras que aminoran la anarquía del sistema internacional) como el incremento de sus relaciones de interdependencia: aspectos que han determinado al desarrollo del CyR en el espacio europeo hasta el momento. En ese sentido, en este artículo se usa como referente teórico de análisis una perspectiva liberal-institucionalista de relaciones internacionales; como mencionan Robert O. Keohane y Joseph S. Nye: "Interdependencia, en su definición más simple, significa dependencia mutua. En política mundial, interdependencia se refiere a situaciones caracterizadas por efectos recíprocos entre países o entre actores en diferentes países" (Keohane y Nye, 1988, p. 22).

En la actualidad, las relaciones China-UE se han caracterizado por el desarrollo de una interdependencia económica en la que prevalece un interés de tipo pragmático. Esto significa que, durante la última década (a partir del inicio de la crisis económica mundial desencadenada entre 2007-2008), las autoridades europeas han preferido subordinar la agenda de la promoción de sus valores normativos (identificados con los principios de la buena gobernanza), en la relación sino-europea, con la prioridad de no obstaculizar el flujo de las relaciones 
comerciales, así como de las inversiones y la cooperación financiera (inversiones tanto de los capitales europeos en China como, de manera significativa, de las inversiones de capital chino en la UE, las cuales se han incrementado en los últimos años). Para la investigación es necesario tomar en cuenta las características estructurales de la relación bilateral actual (las asimetrías normativas y económicas existentes y el pragmatismo económico) con la finalidad de analizar las características de los proyectos de inversión del CyR en la UE. ${ }^{3}$

Como parte de las características estructurales de la relación sino-europea se identifica un conjunto de asimetrías en los aspectos normativos, las cuales representan las principales limitantes para la estructuración de una agenda bilateral común. De la misma manera, a las asimetrías de naturaleza política se agregan otras asimetrías propias de las relaciones económicas, especialmente en dos temas de la agenda bilateral: el déficit comercial que la UE mantiene con China (el cual se ha incrementado de forma constante desde 2001) y la negativa de la UE a otorgar a China el Estatus de Economía de Mercado (EEM), de acuerdo con las normativas de la Organización Mundial del Comercio (OMC).

El trabajo se divide en tres partes. En la primera, se analizan las características estructurales de la relación China-UE en términos políticos y económicos. En la segunda, se analizan las características políticas e institucionales que posee la instrumentación de los proyectos del CyR en la UE en la actualidad. Finalmente, en la tercera, se describe la instrumentación de los proyectos del CyR en el espacio europeo: con fines metodológicos, en la investigación se considera que el gobierno chino ha divido a la UE en diversas áreas que resultan prioritarias para el desarrollo de los proyectos de las dos vertientes en las que se divide el CyR: el Cinturón Económico de la Ruta de la Seda (CERS) y la Ruta de la Seda Marítima del Siglo 21 (RSM-21).

\section{La Iniciativa Cinturón y Ruta de la Seda y las características básicas de la relación China-UE: Política y economía}

Con la finalidad de elaborar un análisis de la instrumentación de los proyectos del CyR en la UE, deben tomarse en cuenta las características básicas de la

3 Como señalan Keohane y Nye sobre la existencia de asimetrías en una relación interdependiente: "Son las asimetrías en la dependencia lo que más probablemente proporcionará fuentes de influencia a los actores en sus vínculos con los demás. Los actores menos dependientes a menudo se encuentran en situación de usar las relaciones interdependientes como fuentes de poder en la negociación sobre un tema y tal vez hasta incidir en otras cuestiones" (Keohane y Nye, 2005, p. 105). 
relación sino-europea, en lo político y lo económico, existentes en la actualidad. En el aspecto político, resaltan algunas semejanzas especialmente orientadas al fomento del multilateralismo como un instrumento para estructurar mecanismos de diálogo y cooperación (recientemente ha sobresalido el consenso sino-europeo que reivindica el respeto a las normativas de la OMC y al Acuerdo sobre el Cambio Climático de París, 2015). Así mismo, se presentan profundas asimetrías entre las preferencias normativas existentes entre China y la UE, tal es el caso de la agenda de la buena gobernanza europea que incluye temas como la promoción de la democracia, la protección de los derechos humanos y la trasparencia: algo que provoca tensiones en la relación, debido a que el gobierno chino podría considerar algunos de los temas de la agenda europea como intervencionistas en sus asuntos internos (Rocha Pino, 2018). En el aspecto económico, sobresale la importancia de los intercambios comerciales de la relación sino-europea, al mismo tiempo que ambos actores mantienen disputas en sus relaciones económico-comerciales, entre las cuales resultan prioritarios dos temas en la agenda: 1) el déficit comercial estructural desfavorable a la UE y 2) la negativa europea a otorgar el EEM a China.

En la actualidad, también debe considerarse la función que posee el CyR como un instrumento de la diplomacia china para consolidar la expansión de sus intereses a nivel global, pero sin significar, por el momento, una ruptura con el sistema de gobernanza. Como señala Yilmaz Kaplan (2017, p. 9): “...la iniciativa OBOR es el resultado de una deliberación de China sobre la estructura internacional dominada por Occidente, y fue diseñada como un proyecto institucionalista geo-funcional con el objetivo de iniciar un cambio tanto gradual como endógeno en el sistema". El CyR es un reflejo de cómo China acepta su papel para apoyar al sistema de gobernanza del comercio, particularmente para mantener las relaciones de interdependencia económica con los actores occidentales; pero, simultáneamente: "la Iniciativa es una manifestación de que China se reúsa a tener un papel pasivo y desea convertirse en un "emprendedor con una agenda" en el mundo" (Kaplan, 2017, p. 10).

En este momento, los objetivos básicos de China en la instrumentación del CyR se relacionan con la ampliación de los mercados para sus exportaciones y "para apoyar a sus empresas conforme se posicionan de forma ascendente en las cadenas de valor" (Holslag, 2017, p. 53). Por esta razón, en la investigación se considera que los objetivos básicos del CyR pueden ser compatibles con los valores y objetivos del proceso de la integración europea, aunque deben tomarse en cuenta las asimetrías estructurales existentes en la relación China-UE. 


\title{
1.1 Las relaciones políticas China-UE: Entre el pragmatismo económico y las asimetrías normativas
}

\begin{abstract}
Para realizar un análisis de la instrumentación de los proyectos del CyR en la UE, debe tomarse en cuenta una particularidad histórica: este proceso forma parte del incremento de intereses económicos de China en el espacio europeo, y de un aumento de las actividades de inversión del capital chino en las economías europeas, tanto en los ámbitos comercial como financiero. Igualmente, el momento en que los intereses de China se amplían y diversifican en la UE ha coincidido, de forma significativa, con el contexto de la crisis económica mundial iniciada entre 2007-2008.
\end{abstract}

La creciente centralidad de la UE - una organización internacional que por sí misma es una potencia económica- y de China - una potencia en ascenso con una presencia creciente en diversos espacios geopolíticos en todo el globo-, en el sistema internacional, hace necesario describir las características de los proyectos de desarrollo hacia el exterior llevados a cabo por ambos actores, a partir de la generación de sus respectivos intereses y preferencias normativas: como en el resto de sus posturas en política exterior, ambas estrategias presentan algunas similitudes y también diferencias notables.

Por una parte, como menciona David Scott (2009), tanto China como la UE hacen énfasis en la cooperación económica además de mantener preferencias por la importancia de la estabilidad regional y el combate a las amenazas a la seguridad internacional como el terrorismo, el extremismo o el crimen organizado trasnacional. Pero entre ambos actores existen diferencias significativas entre sus preferencias normativas: por un lado, la UE enfatiza la difusión de los valores de la buena gobernanza, la lucha contra la corrupción y el fortalecimiento del Estado de derecho (Kavalski, 2013; Manners, 2009). Por otro lado, China mantiene como prioridades los principios doctrinales de su política exterior basados en sus cinco principios de coexistencia pacífica y en conceptos como la multipolaridad, la "democratización de las relaciones internacionales" y la doctrina del desarrollo pacífico. Los principios de la política exterior de China se relacionan con la no intervención en los asuntos de otros países, la no confrontación con terceros, la defensa de la soberanía y la salvaguarda irrestricta de su integridad territorial (Kavalski, 2013).

Además, China mantiene, como otra prioridad, la continuación de su proceso de modernización (en tanto un proceso histórico), el cual depende de la eficiencia y estabilidad del desempeño económico del régimen del Partido Comunista Chino (PCCh): el funcionamiento de la economía ha sido un factor de legitimidad del régimen desde el inicio del proceso de modernización. Por otra 
parte, la relación China-UE experimentó una modificación en sus preferencias, debido al desarrollo del contexto de crisis económica: a partir del periodo 2009 2010, la difusión de los valores normativos de la UE, en su agenda bilateral con China, sufrió una subordinación en comparación al énfasis en la cooperación económica y comercial.

La instrumentación de una relación pragmática, en términos económicos, impulsada por las mismas autoridades de la UE, caracterizó la agenda bilateral de cooperación y diálogo en la relación China-UE, especialmente durante los años 2010-2014. El documento estratégico que refleja el periodo de aproximación pragmática en la relación sino-europea, durante este periodo es la denominada Agenda Estratégica de Cooperación China-UE 2020 (en adelante Agenda 2020) redactada en 2013 de manera conjunta por el Servicio Europeo de Acción Exterior y el Ministerio de Asuntos Exteriores de China (Servicio Europeo de Acción Exterior y Ministerio de Asuntos Exteriores de China, 2013). Sin embargo, conforme los efectos de la crisis económica en la UE comenzaron a ser superados, las autoridades europeas iniciaron una redefinición de los términos de la relación bilateral enfatizando nuevamente al diálogo sobre cuestiones normativas (especialmente sobre derechos humanos), a partir del inicio del periodo de Jean-Claude Juncker como presidente de la Comisión Europea (ejercida durante el término 2014-2019). ${ }^{4}$

Un ejemplo de lo anterior fue la introducción de un diálogo sobre derechos humanos que acompaña la agenda de la Dirección General de Comercio de la UE en sus relaciones de negociación con China durante la gestión de la Comisaria de Comercio, Cecilia Malmström, a partir de 2015, o las críticas de las autoridades europeas hacia el régimen chino a propósito del fallecimiento del disidente Liu Xiaobo en 2017 (Comisión Europea, 2017a; Juncker, 2015; Malmström, 2015). Las autoridades europeas condicionan su apoyo a la proyección del CyR en la UE a que el gobierno y las empresas de China cumplan con las leyes y normativas comunitarias e igualmente se comporten de manera coherente con los valores europeos de la buena gobernanza. Como señalan Ghiasy y Zhou, la instrumentación de los proyectos del CyR en territorio europeo se presenta como una oportunidad para que la UE pueda involucrar a China "...en el tipo de "orden global basado en reglas" más acorde con sus propios intereses y valores" (2017, pp. x-xi).

4 Con este propósito la Comisión Juncker redactó en 2016 el documento estratégico Componentes de una nueva estrategia de la UE respecto a China (Comisión Europea y Alto Representante de la Unión para Asuntos Exteriores y Política de Seguridad, 2016). Este nuevo documento estratégico de la UE sobre China se adhiere a la agenda de la Estrategia Global sobre Política Exterior y de Seguridad de la UE también formulada en 2016 (Servicio Europeo de Acción Exterior, 2016). A partir del 1 de diciembre de 2019 inició el periodo de Ursula von der Leyen como presidenta de la Comisión Europea y de Josep Borrell como el alto representante para Asuntos Exteriores y Política de Seguridad. 
Como mencionó Jean-Claude Juncker en su discurso durante la 12 Cumbre de Negocios UE-China realizada en Bruselas en junio de 2017:

Tanto la UE como China tienen un interés clave en llevar a cabo la Iniciativa Cinturón y Ruta. Nuestro mensaje es que podemos ayudar a construirla, pero las reglas deben ser las mismas para todos. Las empresas europeas deben tener una buena oportunidad de poder licitar y competir en los mismos términos que sus contrapartes chinas. Debemos asegurar, allí también, un campo de juego equitativo. Me siento muy alentado por las declaraciones de China sobre hacer de Cinturón y Ruta una iniciativa abierta, transparente e inclusiva. (Comisión Europea, 2017b, p. 3)

La redefinición de las relaciones China-UE, ejercitada durante la Comisión Juncker, hasta noviembre de 2019, ocasionó tensiones políticas y normativas en la relación bilateral que no intervinieron, de forma significativa, en el flujo de las relaciones económico-comerciales ${ }^{5}$; sin embargo, este aspecto debe ser tomado en cuenta como un factor que puede entrometerse, de manera recurrente, en el desarrollo de las relaciones China-UE. Un caso relevante de estas tensiones ocurrió durante 2019, conforme el gobierno chino sumaba acuerdos de cooperación con Estados miembros de la UE para la implementación de proyectos del CyR en sus territorios nacionales. Estos acuerdos de cooperación bilaterales son denominados memorandos de entendimiento (MdE) y son acordados con los actores internacionales interesados en participar en los proyectos del CyR (principalmente en obras de infraestructura). En el espacio de la UE, algunos Estados miembros que habían firmado MdE con China eran Hungría, Polonia y Portugal.

En marzo de 2019, el gobierno de Italia accedió a firmar un MdE con China, lo que provocó serias críticas por parte de los gobiernos alemán y francés y del Ejecutivo de la Comisión, debido a la relación de dependencia económica que el gobierno italiano estaba estructurando con China y sus promesas de inversiones (Governo della Repubblica Italiana, 2019). De manera simultánea, la Comisión Europea y el Alto Representante de la UE para la Política Exterior y Asuntos de Seguridad publicaron un Documento Estratégico sobre China en el que denominaban a Beijing como un "socio cooperativo y competidor económico" (Comisión Europea y Alto Representante de la UE para la Política Exterior y Asuntos de Seguridad, 2019).

5 La UE ha aplicado sanciones antidumping en contra de productos chinos; un ejemplo es la aplicación de sanciones compensatorias, por parte de la UE, a las importaciones de acero de origen chino en julio de 2016 (Comisión Europea, 2016a; Comisión Europea, 2016b, pp. 2-4). 
Por su parte, el discurso crítico de la Comisión Europea hacia China facilitó algunos resultados en las siguientes semanas; así, fue iniciado un proceso de negociación de algunos temas económicos y de inversión que se desarrollarían como parte de la agenda de la asociación estratégica durante la 21 Cumbre China-UE realizada en abril de 2019 en Bruselas: un logro de la UE fue que el gobierno chino eliminara las prácticas de transferencia de tecnología forzosa en los casos de la adquisición de activos industriales europeos por empresas chinas (Parlamento Europeo, 2019). Posteriormente, la definición de "socio cooperativo y competidor económico" no alcanzó a ser presentada por la Comisión al Consejo Europeo para ser oficializada como una posición común, manteniéndose así el carácter pragmático en la relación sino-europea. Como menciona Theresa Fallon (2019):

[el Documento Estratégico de 2019] ...nunca recibió el respaldo del Consejo Europeo, lo que significó que los Jefes de Estado y de Gobierno de los Estados miembros de la UE no podían ponerse de acuerdo sobre una posición común más dura sobre China. El documento había solicitado explícitamente este respaldo.

\subsection{La agenda económica en la relación China-UE: El déficit comercial y la cuestión del Estatus de Economía de Mercado de China}

En este apartado se describen dos problemas básicos, los cuales tendrán especial relevancia para el futuro del proyecto CyR en el espacio de la UE: 1) el tratamiento del déficit comercial existente entre China y la UE; y 2) la negativa de los Estados miembros de la UE a otorgar a China el EEM hacia finales del año 2016 (de acuerdo con las normativas de la OMC).

\section{a) El problema del déficit comercial}

Durante 2018, la totalidad del intercambio comercial entre China y la UE ascendió a $€ 604604$ millones. Sin embargo, las relaciones comerciales sinoeuropeas se han caracterizado por el creciente déficit en tanto una asimetría estructural: desde el año 2001, China ha mantenido un superávit favorable en su balanza comercial con los miembros de la UE, de forma ininterrumpida, lo que ha originado protestas por parte de algunos miembros de la UE. Como parte de la relación de interdependencia económica, Bruselas se ha comprometido a continuar ampliando sus relaciones comerciales con China, si se mantienen tres 
condiciones: 1) China debe tener un comercio justo; 2) el respeto a los derechos de propiedad intelectual; y 3) China debe respetar las normativas de la OMC (Steinbuka, Muravska y Kuznieks, 2017).

Desde la perspectiva de la Comisión Europea (2006), la preocupación por el déficit se ha debido a las prácticas comerciales "injustas" mantenidas por la parte china. Con la finalidad de enfrentar el problema del déficit comercial en 2006 la Comisión publicó un documento estratégico sobre China denominado UE - China: Una asociación más fuerte con mayores responsabilidades. Las exigencias europeas para que el gobierno chino se comprometiera a instrumentar medidas para reducir el ritmo de su superávit se convirtieron en una prioridad, a partir de 2006, y las primeras muestras sobre una reducción real del déficit comercial europeo se comenzaron a evidenciar a partir de 2009 (Consejo Europeo, 2006; Dirección General de Comercio de la Comisión Europea, 2018; Reuter, 2007).

Sin embargo, la cuestión de la balanza comercial ha continuado siendo un aspecto nodal en la relación bilateral y, en el presente, debe considerarse una variable esencial para la aceptación o el rechazo de las economías europeas de los proyectos del CyR en la UE. Desde 2002, el déficit comercial en la relación China-UE ha aumentado hasta $€ 185001$ millones, el equivalente al 1 $\%$ del producto interno bruto de la UE: "la ampliación del déficit comercial bilateral refleja un efecto base: ha sucedido a pesar de que las exportaciones de la UE a China han crecido más rápidamente que las exportaciones de China a la UE" (Dadush, Domínguez-Jiménez y Gao, 2019, 6). La cuestión del déficit comercial es relevante, si se considera que la Iniciativa Cinturón y Ruta consiste en un conjunto de proyectos para la construcción de infraestructura, con la finalidad de incrementar los flujos comerciales entre los actores que se integren a estos mismos: en el espacio europeo existe una preocupación alrededor de que el incremento del comercio solo podría elevar más su déficit comercial con China. 
Tabla 1.

Relaciones comerciales Unión Europea-China (2012-2018) (en millones de euros)

\begin{tabular}{|c|c|c|c|c|}
\hline Año & $\begin{array}{c}\text { Exportacioneas Unión } \\
\text { Europea-China }\end{array}$ & $\begin{array}{c}\text { Importaciones Unión } \\
\text { Europea-China }\end{array}$ & $\begin{array}{c}\text { Balanza } \\
\text { comercial }\end{array}$ & Total \\
\hline 2012 & 144227 & 292128 & -147894 & 436350 \\
\hline 2013 & 148115 & 280119 & -132004 & 428234 \\
\hline 2014 & 164679 & 302490 & -137810 & 467169 \\
\hline 2015 & 170361 & 351044 & -180683 & 521405 \\
\hline 2016 & 169744 & 350250 & -180506 & 519994 \\
\hline 2017 & 197920 & 375201 & -177281 & 573121 \\
\hline 2018 & 209846 & 394847 & -185001 & 604604 \\
\hline
\end{tabular}

Nota: Dirección General de Comercio de la Comisión Europea, 2018 y Eurostat, 2020.

Para Holslag, el CyR representa un "enorme reto" para Europa: se contextualiza en un momento en que las exportaciones europeas hacia las economías euroasiáticas se encuentran en un momento de estancamiento. Durante el período 2008-2014 "las exportaciones europeas hacia los países de la Nueva Ruta de la Seda disminuyeron en USD 25 mil millones, mientras que las exportaciones chinas aumentaron en USD 250 mil millones" (Holslag, 2017, p. 56). La disminución de los productos europeos en el espacio euroasiático fue mayor en el sector de la alta tecnología, en la que se observa un aumento de los productos tecnológicos fabricados en China. Desde la perspectiva de dicho autor, si la Nueva Ruta de la Seda resulta exitosa, las pérdidas comerciales serán mayores en el largo plazo.

A las pérdidas comerciales, se agrega la disminución de la influencia política de la UE (es decir, de la proyección de su poder blando) en la diversidad de países euroasiáticos. Sin embargo, la instrumentación de los proyectos de conectividad del CyR, entre China y la UE, igualmente posee el potencial de incrementar los flujos comerciales en la relación sino-europea, debido al aumento de la eficacia de las redes de transportación y la disminución de sus costos: un nuevo contexto que podría ser benéfico para las exportaciones europeas; como señalan Richet, Ruet y Wang (2017, pp. 129-130):

Por un lado, la exportación de bienes de China hacia Europa puede tener un impacto negativo sobre las exportaciones netas de Europa. Sin embargo, esto dependerá sobre si existe un potencial todavía no explotado para los productos chinos en los mercados europeos... Por otro lado, investigaciones 
recientes muestran que la demanda china se orienta cada vez más hacia los productos extranjeros, que a los productos nacionales, y esto probablemente aumentará las importaciones chinas provenientes de Europa.

Como mencionan estos autores, conforme se instrumenten los proyectos del CyR, será importante que las economías europeas insistan en una reciprocidad en su acceso al mercado interno de China.

\section{b) La cuestión del EEM}

La negativa de los miembros de la UE para otorgar el EEM a China se relaciona directamente con la existencia del déficit comercial desfavorable para la parte europea. Como menciona Feng (2008), si bien el incremento del déficit comercial en las relaciones sino-europeas es un problema que ha causado mucha preocupación entre los Estados miembros de la UE, estos no han tomado medidas para impedir la entrada de productos chinos en el Mercado Común (al mismo tiempo que las economías europeas se han beneficiado aumentando sus exportaciones a China, aunque sin reducir el déficit). Por lo contrario, en lugar de impedir el acceso de productos chinos a la UE, las autoridades europeas han solicitado a China la apertura de ciertos sectores de su economía (especialmente del sector servicios y las telecomunicaciones) para las compañías europeas: "el problema en buena medida no se debe al déficit comercial sino al acceso del mercado" (Feng, 2008, pp. 77-78); sin embargo, China teme la apertura de su sector de servicios, debido a que su productividad es más baja frente las empresas europeas.

Por su parte, China ha solicitado a la UE el reconocimiento de su estatus como una economía de mercado, con lo cual espera disminuir las investigaciones antidumping que se realizan en contra de sus productos. La intervención del Estado chino en las actividades productivas y la debilidad del Estado de derecho han sido los argumentos de la UE para negar el EEM a China. En el primer caso, debido a que las empresas de propiedad estatal china reciben numerosos subsidios del Estado, así como un trato preferencial para el abastecimiento de ciertas materias primas. Al mismo tiempo, dichas empresas reciben un trato tributario preferencial: algunas empresas estatales chinas pueden sobrevivir a sus propias crisis internas financieras o de productividad, debido a la ayuda del Estado, lo que provoca una "distorsión" en el funcionamiento del mercado (Rémond, 2007).

Por otra parte, los desacuerdos en materia comercial por el déficit y la cuestión del EEM no significan un obstáculo definitivo para la continuidad de las relaciones de cooperación en las áreas de desarrollo científico y tecnológico entre 
China y los países de la UE. Si bien continúan las protestas de muchas empresas europeas, dedicadas a las manufacturas, por el mal uso de sus marcas y por las violaciones a sus derechos de propiedad intelectual (DPI), que se hacen en China, en el caso de la cooperación en tecnología avanzada la situación es diferente (Li, 2016).

En la actualidad, el marco legal básico para regular la cooperación sino-europea, en el campo científico y tecnológico, es el Acuerdo de Cooperación Económica y Comercial China-Comunidad Económica Europea de 1985 y la Asociación para la Cooperación Científica y Tecnológica China-UE de 2009 (Consejo Europeo, 2009; Hong, 2017) ${ }^{6}$. La UE es el socio más importante de China en el campo de la transferencia de tecnología, el acceso al mercado chino, por parte de las empresas europeas, es el principal incentivo para la cooperación tecnológica. Como menciona Nicola Casarini (2015, p. 124), la transferencia tecnológica ocurre en unos términos que estas empresas no aceptarían con otros actores internacionales "en la mayoría de los casos" (teniendo como excepciones a la cooperación transatlántica y a Japón).

La continuidad de una agenda en la cooperación científica y tecnológica sinoeuropea contribuye a explicar el rechazo de la UE a aplicar una política de veto a las actividades comerciales de las empresas de tecnología digital chinas (como Huawei y ZTE) en el espacio europeo (al contrario de la política de sanciones proteccionistas instrumentada por el gobierno de Donald Trump en Estados Unidos y su prohibición al internet $5 \mathrm{G}$ de las empresas chinas en su país). En este rubro, la UE se ha enfocado a exigir, a las empresas tecnológicas chinas, que se ajusten, de forma estricta, a las regulaciones comunitarias: esta es una condición imprescindible para la proyección de la denominada "Ruta de la Seda Digital" en Europa (China File, 2019).

\section{La proyección del CyR en la Unión Europea: Aspectos políticos e institucionales}

A continuación, se analiza la importancia del apoyo de la UE a la implementación del CyR en el espacio europeo y sus características institucionales.

6 El Acuerdo de Cooperación Económica y Comercial China-Comunidad Económica Europea de 1985 es el tratado comercial referencial que continúa determinando las directivas básicas de las relaciones comerciales entre China y la UE hasta la actualidad (China y la UE no han podido negociar un nuevo acuerdo comercial que sustituya al Acuerdo de 1985). 


\subsection{El marco institucional para la proyección del CyR en la UE}

Una característica primordial en la proyección del CyR en el espacio euroasiático, tal como es planeado actualmente por el gobierno chino, reside en que requiere del apoyo político y económico de la UE hacia la instrumentación de sus proyectos de infraestructura (tanto por parte del nivel supranacional como de los Estados miembros de la UE).

Como señalan Ghiasy y Zhou (2017), dicho apoyo europeo se considera una condición necesaria para que la iniciativa china pueda contribuir, de manera efectiva, a la conformación de un mercado de bienes y servicios integrado en Eurasia. En el caso específico del CyR-CERS, la iniciativa necesita el apoyo de dos regiones fundamentales en Eurasia: por un lado, de la UE (teniendo a la asociación estratégica China-UE y al Foro de Cooperación China-Europa Central y Oriental también denominado Foro $17+1-$ como dos plataformas que pueden funcionar como instituciones coordinadoras de la agenda de actividades) y, por otro lado, el apoyo de los países de Asia Central (en este caso, con la Organización de Cooperación de Shanghai como una plataforma institucional con una trayectoria que se remonta a 2001, por lo menos). A través del CyR, el gobierno chino ha mostrado un interés creciente para participar en construir o reconstruir porciones de la infraestructura europea de comunicaciones, en especial equipamientos, aeropuertos, puertos, línea férreas y carreteras: "si el CyR tiene éxito transformaría el panorama político y económico en Eurasia" (Le Corre y Pollack, 2017, p. 7). Como mencionan Philippe Le Corre y Jonathan Pollack (2017), existe una similitud entre las inversiones de China en dos regiones necesitadas de recursos y de la renovación (por lo menos) de sus servicios de infraestructura de comunicaciones como Asia central y Europa Central y Oriental.

Además de la cooperación económica sino-europea, Ghiasy y Zhou (2017) consideran otros cuatro objetivos básicos en la necesidad de China para involucrar a la UE en el CyR:

1. China considera a la UE como un socio potencial para el mantenimiento de la paz y la estabilidad en Eurasia (al mismo tiempo que la UE puede funcionar como un contrapeso frente al poder de Estados Unidos).

2. El CERS puede contribuir a acelerar las relaciones de interdependencia económica entre la UE y otros socios económicos ubicados en Asia del Este (algo que podría aminorar los lazos de dependencia en la relación transatlántica, especialmente tras la suspensión de las negociaciones de la Asociación Transatlántica de Comercio e Inversiones (TTIP, por sus siglas en inglés) por parte del gobierno de Donald Trump en enero de 2017). 
3. Iniciar un proceso más ambicioso, en el escenario de una nueva relación sino-europea: “...la centralidad económica y política del mundo podría recalibrarse desde los bloques económicos transatlántico y transpacífico hacia Eurasia continental" (Ghiasy y Zhou, 2017, p. 10), es decir, al constituir una mega región euroasiática, en la que además existe otro proyecto de integración en el caso de la Unión Económica Euroasiática (UEEA) (Diaz Barrado, 2016).

4. Una mayor diversificación de las relaciones económicas y financieras de China con la UE podría, igualmente, reducir la dependencia en sus relaciones económicas con Estados Unidos y disminuir los riesgos ante posibles sanciones y guerras comerciales en la relación sinoestadounidense. Por su parte, Mario Esteban y Yuan Li (2017) coinciden en que la instrumentación del CyR puede provocar una modificación en las relaciones de dependencia económica entre China y sus principales socios económicos (como Estados Unidos).

De acuerdo con Le Corre y Pollack (2017), las políticas comunitarias de la UE hacia China evidencian un reconocimiento sobre la progresiva centralidad china en el futuro económico del continente. En la actualidad, los niveles de los montos de comercio e inversiones entre China y las principales economías de la UE se aproximan (e incluso exceden) a los existentes en las relaciones transatlánticas (a partir de 2016, China sobrepasó a Estados Unidos como el principal socio comercial de Alemania). Por su parte, China igualmente reconoce la importancia de la UE como uno de sus mayores socios económicos en el espacio euroasiático (incluso con mayores posibilidades de las existentes en la relación sinoestadounidense y sin las desventajas de sus riesgos de seguridad). El avance de esta tendencia puede depender de la capacidad de los aliados transatlánticos para definir su relación bilateral en el contexto del ascenso de China y la diversificación de los proyectos de integración en el espacio euroasiático.

En la actualidad, la relación entre el sistema institucional-legal de la UE con los proyectos del CyR se encuentran regulados, por el lado europeo, por tres documentos: 1) la Estrategia Global de la UE de 2016; 2) el documento estratégico de la UE sobre China de 2016, y 3) el Memorando de Entendimiento sobre la Plataforma de Conectividad China-UE, acordado entre la Comisión Europea y el gobierno chino en 2015 (Comisión Europea, 2015; Comisión Europea y Alto Representante de la Unión para Asuntos Exteriores y Política de Seguridad, 2016; Servicio Europeo de Acción Exterior, 2016). En el caso específico de la plataforma de conectividad, esta se encuentra diseñada "para buscar oportunidades conjuntas de inversión a lo largo de la Ruta de la Seda" (Ghiasy y Zhou, 2017, p. 45). 


\subsection{Características de los planes de inversión del CyR en la UE}

Además de los documentos oficiales, debe mencionarse el inicio de la negociación de un acuerdo bilateral de inversiones (ABI) iniciada por la Comisión Europea y el gobierno chino en 2010: el anuncio sobre el inicio de las negociaciones para concluir un ABI se efectuó durante el desarrollo de una de las peores coyunturas en la crisis económica en la eurozona (la correspondiente al agravamiento de la crisis de deuda en países como Grecia, Portugal y España). Las negociaciones sobre el ABI iniciaron oficialmente en 2013 y en enero de 2016 se había alcanzado la novena ronda de negociaciones sobre el acuerdo (su conclusión se planea para el año 2020) (Comisión Europea, 2016c).

El principal estímulo de los países europeos para concluir la negociación del $\mathrm{ABI}$ es la falta de liquidez para invertir en algunos de los proyectos de infraestructura propuestos: una consecuencia de la existente crisis de endeudamiento $\mathrm{y}$, por lo tanto, de confianza en el sistema financiero europeo. De acuerdo con Steinbuka, Muravska y Kuznieks (2017), en el año 2015, China dirigió $€ 20$ 000 millones en inversión directa en Europa; en ese momento la mayoría de los Estados miembros de la UE habían establecido contactos de alto nivel con su contraparte china para sus relaciones de inversión en el nivel bilateral; de esta manera, la promesa de inversiones de capital chino en algunos países europeos ha generado una expectativa que incrementa la cantidad y la calidad de los contactos sino-europeos "como una zanahoria para otros objetivos de su política exterior". Un ejemplo de este proceso han sido los flujos de inversión hacia los países de Europa central y oriental como parte de los acuerdos de cooperación alcanzados durante las actividades del Foro 17+1. Por su parte, Jones y Liu señalan que las naciones más pobres de Europa central han aceptado los créditos de ayuda del capital chino como una alternativa para recibir inversiones (2017, p. 487).

Sin embargo, más allá de las directivas generales contenidas en estos documentos oficiales, hasta el momento no ha existido una política oficial unificada de la UE hacia el CyR, al mismo tiempo que ha existido "una limitada reflexión interna al interior de la UE sobre las implicaciones a largo plazo del CyR" (Ghiasy y Zhou, 2017, p. 46) por parte de las instituciones europeas encargadas de regular esas relaciones. Esta situación podría atribuirse al carácter poco institucionalizado del CyR; sin embargo, entre algunos actores políticos y económicos, tanto en el nivel supranacional como al interior de los Estados miembros, sí es posible encontrar aproximaciones más unificadas hacia los proyectos del gobierno chino (Ghiasy y Zhou, 2017). 
Los objetivos del CyR parecen ser coherentes con el principio europeo del multilateralismo eficiente (subrayado en la Estrategia Europea de Seguridad de 2003 y reiterado en la Estrategia Global de 2016). En el documento oficial "Visión y Acciones para la Construcción del Cinturón Económico de la Ruta de la Seda y de la Ruta de la Seda Marítima del siglo 21", que contiene las directivas básicas del CyR, se hace la siguiente mención al multilateralismo y el respeto al sistema de economía de mercado y el derecho internacional:

Los países a lo largo de Cinturón y Ruta deberían mejorar la cooperación aduanera, como el intercambio de información, el reconocimiento mutuo de las reglamentaciones y la asistencia mutua en la aplicación de la ley; mejorar la cooperación bilateral y multilateral en los campos de inspección y cuarentena, certificación y acreditación, medición estándar e información estadística; y trabajar para asegurar que el Acuerdo de Facilitación del Comercio de la $\mathrm{OMC}$ entre en vigor y se instrumente. (Comisión Nacional para el Desarrollo y la Reforma, Ministerio de Asuntos Exteriores y Ministerio de Comercio de China, 2015)

De la misma manera, el CyR converge con otras prioridades de la UE como: a) la gobernanza económica global a través de un sistema abierto y basado en el Estado de derecho y b) la importancia de los ordenamientos de cooperación a nivel regional como una forma de gobernar el proceso de conectividad en Asia (un concepto presente en la Estrategia Global de 2016). Como mencionan Ghiasy y Zhou (2017, p. 46), "tal como se propone en el documento Visión y Acciones, China busca metas similares" en teoría el CyR también coincide con las estrategias subregionales de la UE y los programas europeos específicos para cada país, particularmente aquellos que promueven la seguridad humana por medio del desarrollo socioeconómico.

Por otro lado, una de las asimetrías que puede generar mayores conflictos en la instrumentación de los proyectos del CyR en la UE son las diferencias entre los sistemas legales de la UE y de China. Para Richet, Ruet y Wang queda clarificado que la proyección del CyR en los países europeos debe realizarse mediante un respeto estricto de la legislación europea y sus normativas (2017). Por su parte, China mantiene una relación pragmática con el derecho internacional (un ejemplo son las normativas de la OMC y de su Sistema de Resolución de Disputas, las cuales son respetadas por China como un marco legal referencial del derecho internacional). La razón de este comportamiento es clara: por un lado, China prefiere continuar con un sistema normativo homogéneo (al menos para todos los países miembros de la OMC), que enfrentarse con una diversidad de sistemas legales y normativos (correspondientes a la diversidad de países en los cuales podrían instrumentarse los proyectos del CyR). En este momento de su desarrollo económico, a China parece no interesarle efectuar una ruptura del 
sistema de gobernanza del comercio global (el sistema actual ha sido funcional para el proyecto de modernización de China, tal como lo esclareció Xi Jinping durante su discurso ante el Foro de Davos de 2017) (Xi, 2017).

La consolidación de una relación sino-europea basada en el pragmatismo económico, favorecida por los actores con la finalidad de evitar una ruptura de sus intercambios comerciales, de inversión y cooperación tecnológica, probablemente haya sido una de las consecuencias de mayor significación del periodo de la crisis global para los vínculos bilaterales (un proceso en el que China también ha aprovechado la debilidad económica de la UE durante el periodo de crisis, especialmente durante 2010-2012). Como se ha comentado, a pesar de la existencia de ciertas tensiones, producto de sus asimetrías políticas, China y la UE han incrementado sus relaciones de interdependencia económica durante el periodo 2012-2018 (Cfr. Tabla 1). Otra evidencia del consenso entre China y la UE por intensificar sus relaciones de interdependencia económica puede ser la multiplicación de sus diálogos de cooperación económica y sectorial, que forman parte de la agenda de la asociación estratégica China-UE, los cuales alcanzan, en la actualidad, a más de 50 diálogos que abordan diversas temáticas y mantienen actividades a lo largo del año (Hong, 2017).

En el ámbito de la instrumentación de los proyectos del CyR en Europa, el gobierno chino se apoya en un discurso que presenta a la iniciativa como complementaria a los planes europeos para desarrollar infraestructura e impulsar la conectividad al interior de Europa y entre el espacio europeo y "más allá" (Richet, Ruet y Wang, 2017, p. 98). Este espacio integrado abarca al resto del espacio euroasiático enlazando a la costa oriental de China con las regiones de frontera situadas entre las provincias occidentales chinas (Gansu, Xinjiang, y Tibet) con Asia Central y, a su vez, alcanza a Europa Oriental y Occidental. De acuerdo con Ghiasy y Zhou (2017, p. 46), el grado de compatibilidad entre el CyR y los intereses, y prioridades de la UE dependerá del "carácter y la calidad de la instrumentación de los proyectos de China" en interacción con el conjunto de riesgos de la gobernanza y las tensiones geopolíticas existentes (como riesgos a la seguridad no convencionales o la inestabilidad política en algunos actores).

Así mismo, en el espacio europeo todavía debe ser evaluado el impacto real de la instrumentación de los proyectos del CyR y su efecto real sobre las preferencias europeas hacia los valores de la buena gobernanza: de forma ideal, la participación de la UE en los proyectos del CyR puede convertirse en un vehículo para la promoción de los valores europeos; sin embargo, dicha posibilidad debería proyectarse sin demasiado optimismo, recordando la experiencia de la promoción de los valores europeos entre los países exsoviéticos de Asia Central o en la misma China. En el aspecto pragmático, es claro el interés de algunos 
sectores empresariales europeos para participar en los proyectos del CyR en la UE y otras regiones de Eurasia (Comisión Europea, 2017b).

\section{La proyección del CERS en la UE}

El inicio de la instrumentación oficial de los proyectos del CyR en la UE se puede identificar con la visita de Estado realizada por Xi Jinping a Alemania y Bélgica, en marzo de 2014; previamente, en abril de 2013, se había acordado la Agenda 2020; sin embargo, dicho documento no incluyó ningún plan de acción concerniente a los proyectos del CyR. Durante su visita a Alemania en 2014, Xi visitó las inversiones chinas realizadas en el puerto ferroviario de Duisburgo, el cual es la conclusión del ferrocarril Yuxinou (Chongqing-Duisburgo) (Richet, Ruet y Wang, 2017).

Hasta el momento, el CERS se compone de seis rutas que se encuentran en la etapa de planeación o de construcción: el Corredor China-Mongolia-Rusia; el Nuevo Puente Terrestre Euroasiático; el Corredor China-Asia Central-Asia Occidental (incluyendo a Irán); el Corredor Económico China-Pakistán; el Corredor de la Península de Indochina y el Corredor Bangladesh-China-India-Myanmar (BCIM). El Nuevo Puente Terrestre Euroasiático recorre hacia el espacio europeo conectando a la ciudad de Chongqing vía la ciudad de Xian (a través de Asia Central) con Duisburgo y Rotterdam y a Chengdu con la ciudad de Lodz (en Polonia) vía Kazajistán, Rusia y Bielorrusia. La ciudad de Horgos (en Xinjiang), ubicada entre la frontera sino-kazaja, será suplementada con infraestructura logística, servicios bancarios y facilidades industriales (Gonçalves, 2017).

De acuerdo con Richet, Ruet y Wang (2017), el comportamiento de las inversiones del CyR en la UE indica una división jerárquica del espacio europeo de acuerdo con los intereses prioritarios del gobierno chino; de la misma manera, el impacto del CyR tiene una significación diferente para cada uno de los miembros de la UE (Esteban y Yuan, 2017). Las áreas de inversión del CERS en la UE, por orden de prioridad, son las siguientes:

1. El área de mayor prioridad para el CERS, en Europa, es a la que pertenecen los países de Europa Central y Oriental, debido a que esta región enlaza a los proyectos del CyR, que se desarrollan actualmente en las regiones de frontera entre las provincias occidentales de China con Asia Central, con los proyectos del CyR orientados hacia el espacio europeo en general: los países de Europa Central y Oriental son el puente entre Eurasia Central y Europa Occidental. 
Con la finalidad de incrementar sus relaciones de cooperación con esta región, desde 2012 el gobierno chino ha impulsado la conformación del Foro de Cooperación China-Europa Central y Oriental. El Foro 17+1 incluye a 17 países de Europa Central y Oriental (de los cuales doce países son miembros de la UE) y, si bien es patrocinado por China, sus actividades no se encuentran coordinadas por la Comisión Europea (la instancia responsable de la coordinación de la agenda de la asociación estratégica China-UE).?

Los proyectos de cooperación negociados durante las actividades del Foro $17+1$ pueden tener una modalidad tanto multilateral como bilateral (entre China y los países interesados); en la actualidad dicho Foro se convierte en una parte fundamental de la coordinación de los proyectos del CERS en los Balcanes y Europa Central. Sin embargo, igualmente puede generar tensiones institucionales en el funcionamiento de la UE: las actividades de cooperación en el Foro 17+1 también son percibidas, por algunos funcionarios en Bruselas, como un riesgo para los principios de coherencia y consistencia en el seguimiento de las directivas de la UE por parte de algunos Estados miembros y la relación que estos mantienen con China: "algunos funcionarios europeos consideran que el actual formato es una amenaza para la cohesión interna y puede promover la construcción potencial de un lobby pro-China en Bruselas" (Ghiasy y Zhou, 2017, p. 46).

En la actualidad, en los Balcanes sobresale la construcción del tren de alta velocidad Atenas-Belgrado-Budapest que conecta al CERS con el extremo de la RSM-21, en el sur de Europa, ubicado en el Puerto del Pireo en Grecia. El ferrocarril está financiado en $85 \%$ por capital chino (el Export-Import Bank of China ha otorgado préstamos a los gobiernos de Hungría y Serbia) (Richet, 2018). En el caso de Polonia sobresale la ruta del mencionado ferrocarril Chengdu-Lodz, en operación desde 2013.

2. La segunda área prioritaria en la instrumentación del CERS es Europa Occidental, espacialmente en el caso de Alemania: en 2017 el intercambio comercial sino-alemán alcanzó € 187977 millones, lo que significó más de un tercio del comercio total entre China y la UE (Statistisches Bundesamt, 2018). En esta área también se incluyen las principales economías europeas como España, Francia, Italia, Países Bajos y Reino Unido (el caso de Reino Unido es especial, debido al alto interés de China por mantener relaciones de cooperación con el sistema financiero de aquel país).

7 Los doce países miembros del Foro 17+1 que pertenecen a la UE son: Bulgaria, Croacia, Eslovaquia, Eslovenia, Estonia, Grecia, Hungría, Lituania, Letonia, Polonia, la República Checa y Rumania. Por otra parte, los cinco miembros europeos del Foro que no pertenecen a la UE son: Albania, Bosnia y Herzegovina, Macedonia del Norte, Montenegro y Serbia. 
Los principales ferrocarriles que actualmente recorren las rutas entre China y los países occidentales de la UE son seis: 1) el ferrocarril Yuxinou que recorre la ruta Chongqing-Duisburgo; 2) el ferrocarril Yixinou que recorre la ruta entre Yiwu-Madrid; 3) el tren Changsha-Hamburgo; 4) el tren HarbinHamburgo; 5) el tren Hefei-Hamburgo; 6) el tren Zhengzhou-Hamburgo. En abril de 2016 se inauguró una ruta de ferrocarril entre Wuhan-Lyon y en enero de 2017 entre Yiwu-Londres (Pomfret, 2018, pp. 5-6).

3. La tercera área son los países en los cuales el gobierno chino, hasta el momento, no ha anunciado proyectos del CERS como los escandinavos. Sin embargo, existe un claro interés del gobierno chino en incluir a las regiones del norte de Europa (particularmente a los países árticos europeos) en algunos proyectos integrados en el CyR, tal como lo evidencia el documento "Una política de China para el Ártico", publicado por el Consejo de Estado de China en enero de 2018 (Consejo de Estado de China, 2018). En este sentido, la nueva prioridad del gobierno chino ha sido denominada de manera informal como la Ruta de la Seda Polar: un proyecto con potencial hacia el futuro, pero del cual solo se conocen sus primeras características y que depende de algunos fenómenos climáticos, tal es el caso del deshielo de algunas regiones árticas como el denominado Paso del Noroeste (Liu, 2018).

\section{La Ruta de la Seda Marítima del siglo 21 en el espacio europeo: el caso del Puerto del Pireo}

En lo concerniente a la RSM-21, las áreas prioritarias se dividen en dos:

1. La primera área es el Mediterráneo Oriental, especialmente en el caso del Puerto del Pireo en Grecia: dicho puerto ha recibido la mayor cantidad de inversiones para el desarrollo de los proyectos del CyR en Europa Oriental y, al mismo tiempo, puede considerarse el punto de encuentro entre el CERS y la RSM-21.

2. La segunda área en orden de prioridad es el Mediterráneo Occidental (debido a las inversiones de capital chino en algunos puertos como en Génova y Valencia) y las inversiones en puertos de la costa atlántica europea como Havre y Rotterdam. Sin embargo, para algunos autores estas inversiones podrían provocar una distorsión en la economía europea, al iniciar un proceso de competencia entre algunos puertos del norte de Europa con las inversiones chinas en los puertos del Mediterráneo como el Pireo. 


\subsection{El Puerto del Pireo y el enlace CERS-RSM-21}

Un caso significativo de inversión de capital chino en el espacio europeo ha sido la concesión del Puerto del Pireo, en Grecia, a la compañía naviera Chinese Ocean Shipping Group Company con sede en Beijing (en adelante COSCO, por sus siglas en inglés). Como parte de las necesidades del gobierno griego por encontrar financiamiento, en 2011 decidió la venta de un conjunto de activos de propiedad gubernamental entre los que se encontraba la mayor parte del Puerto del Pireo (Brown, 2012). El Puerto del Pireo se ha convertido en la principal base para el desarrollo de los proyectos de la iniciativa Cinturón y Ruta en el sur de Europa: al mismo tiempo, el Pireo es el espacio geopolítico en el que se unen el CERS y la RSM-21.

Tras las negociaciones, en el verano de 2011, el gobierno griego otorgó a COSCO la concesión de sus activos en dicho puerto durante un periodo de 35 años, a cambio de USD 5 mil millones aproximadamente, además del compromiso de COSCO para invertir en el puerto un total de USD 700 millones. La concesión lograda en el Puerto del Pireo resultó estratégica para los intereses comerciales de China en Europa: "Los grandes montos de inversión que China exporta a la UE significan que los puertos son de un interés significativo para ese país y que el Pireo en particular tiene un valor estratégico, siendo un competidor potencial para Rotterdam" (Brown, 2012, p. 83).

La empresa COSCO adquirió el $67 \%$ de las operaciones en el Puerto del Pireo en abril de 2016 a un costo de $€ 368$ millones, iniciando así una nueva etapa en el proyecto chino de construcción de infraestructura en los Balcanes: ahora el gobierno chino planea la construcción de una línea de alta velocidad en los Balcanes con la intención de unir al Puerto del Pireo con Europa Central (Reuters, 2016). Para Grecia, el aumento de estas actividades de inversión provocó que su relación con China (previamente poco significativa) se convirtiera en una de las prioridades de la política exterior griega (algo que contó con el apoyo de importantes sectores de la economía griega, como las empresas constructoras navieras) (Huliaras y Petropoulos, 2014): la intensificación de los contactos (durante los gobiernos de Andonis Samarás y Alexis Tsipras) fue producida por la necesidad del gobierno griego por contar con fuentes de financiamiento, para lo cual instrumentó un amplio plan para la privatización de bienes públicos, en este caso el Puerto del Pireo.

No obstante, los términos de la nueva relación sino-griega se han mantenido en el límite del pragmatismo económico: Grecia no ha deseado debilitar su relación con sus socios occidentales (en la UE y en la OTAN) al encontrar a un nuevo socio económico (las inversiones de capital chino) y, por su lado, China 
tampoco ha pretendido interferir en los términos de las relaciones de Grecia con sus socios europeos. Durante la grave crisis política y financiera en Grecia, entre los meses de junio y julio de 2015, el primer ministro Li Keqiang declaró, en Bruselas, su apoyo al mantenimiento de la estabilidad económica en la eurozona, en tanto que el gobierno chino era un acreedor "responsable" de bonos de deuda soberana de Grecia (Dokos, 2012; Li, 2016 ). ${ }^{8}$

Como menciona Dokos (2012, p. 9): "mientras la presencia de China en el Mediterráneo permanezca básicamente económica y no cause fricciones con los socios occidentales de Grecia, Atenas no enfrentará dilemas difíciles". Igualmente, el gobierno de centroderecha encabezado por Kyriakos Mitsotakis, que sucedió al de Alexis Tsipras, luego del proceso electoral de 2019, ha mantenido una relación cercana con Beijing continuando con el pragmatismo económico de la relación bilateral (Psaropoulos, 2019). La inversión china en el puerto griego ha significado contar con una plataforma que facilita la exportación y difusión de los productos chinos en Europa; en este caso, se combinó la necesidad de China por tener una plataforma exportadora para sus productos con la necesidad de un gobierno altamente necesitado de capital, como el griego en el contexto de la crisis de deuda.

\section{Consideración final}

Como parte del proceso de interdependencia económica entre los actores, en el trabajo se identifica que, para China, es necesario el apoyo de la UE para la instrumentación del CyR. Los objetivos básicos del CyR no se encuentran en contradicción con los valores europeos relacionados con el liberalismo económico y con los valores de la buena gobernanza (en un principio), por lo cual ambos actores han podido establecer negociaciones para la instrumentación de algunos proyectos de inversión relacionados con el CyR en la UE. Además, China y la UE han podido acordar documentos conjuntos con la finalidad de regular dichas actividades (como la Plataforma de Conectividad China-UE y el Plan de Inversiones China-Europa).

Las contradicciones existentes en la proyección del CyR en el espacio europeo se relacionan con algunas características estructurales presentes en la relación China-UE, en lo general: en la investigación se analizaron las características más relevantes de estas diferencias, las cuales residen en un conjunto

8 Esta crisis fue provocada por el gobierno del primer ministro Alexis Tsipras, al convocar a un referéndum el 5 de julio de 2015, sobre la aceptación o no, de las condiciones de ajuste económico impuestas en ese momento por la denominada troika (la UE, el Fondo Monetario Internacional y el Banco Central Europeo). 
de asimetrías normativas (la renuencia de China a aceptar la inclusión de los temas de la buena gobernanza en la relación bilateral), económicas (en cuestiones como el déficit comercial y la negativa europea a otorgar el EEM a China) y legales (como las profundas diferencias entre los sistemas legales europeo y chino y su trascendencia para las prácticas de negocios que pueden efectuar algunos inversionistas de origen chino en la UE).

De la misma manera, a pesar de las asimetrías existentes, China y la UE han podido desarrollar un proceso de institucionalización de su relación bilateral mediante las actividades de la asociación estratégica China-UE. Esta asociación estratégica podría desempeñar una función cardinal como una plataforma coordinadora de las actividades de cooperación y para la gestión de las asimetrías existentes. La relación sino-europea se ha estructurado mediante la lógica de una relación de interdependencia, que tiene como prioridad el desarrollo de sus relaciones económicas y comerciales (a pesar de las asimetrías existentes): estas prioridades resultan el principal incentivo para que China y la UE continúen negociando las asimetrías y los desacuerdos en la relación bilateral. La proyección del CYR en el espacio europeo dependerá del interés pragmático con el que ambos actores consideren que pueden obtener ganancias mutuas de sus proyectos conjuntos.

\section{Referencias}

Brown, K. (2012). China's Overseas Investment in the European Union. The International Spectator, 47(2), 74-86.

Casarini, N. (2015). China's Rebalancing towards Europe. The International Spectator, 50(3), 122-129.

China File. (18 de octubre, 2019). The Future of Huawei in Europe (A China File Conversation). http://www.chinafile.com/conversation/future-of-huawei-europe

Comisión Europea y Alto Representante de la UE para la Política Exterior y Asuntos de Seguridad. European Commission and HR/VP Contribution to the European Council. EU-China (2019). A Strategic Outlook (JOIN 5 final). https://ec.europa.eu/commission/sites/beta-political/files/communicationeu-china-a-strategic-outlook.pdf

Comisión Europea. (2017a). Joint statement by the President of the European Commission, Jean-Claude Juncker, and the President of the European Council, Donald Tusk on the Passing Away of Liu Xiaobo. http://europa.eu/rapid/ press-release_STATEMENT-17-2032_en.htm. 
Comisión Europea. (2017b). Speech by President Jean-Claude Juncker at the 12th EU-China Business Summit. http://europa.eu/rapid/ press-release_SPEECH-17-1526_en.htm.

Comisión Europea. (2016a). 18th EU-China Summit in Beijing (Announcement). http://www.europa.eu/rapid/press-release_AC-16-3700_en.pdf.

Comisión Europea. (2016b). Comunicación de la Comisión al Consejo Europeo y al Parlamento Europeo. Hacia una política comercial sólida para la Unión Europea, en beneficio del empleo y el crecimiento (COM 690 final). http:// ec.europa.eu/transparency/regdoc/rep/1/2016/ES/COM-2016-690-F1-ESMAIN.PDF

Comisión Europea. (2016c). EU and China agree on scope of the Future Investment Deal. http://trade.ec.europa.eu/doclib/press/index.cfm?id=1435.

Comisión Europea. (2015). Investment Plan for Europe goes global: China announces its contribution to \#investEU. http://europa.eu/rapid/ press-release_IP-15-5723_es.htm

Comisión Europea. (2006). EU - China: Closer partners, growing responsibilities (COM 631 final). http://eurlex.europa.eu/LexUriServ/LexUriServ.do?uri=C OM:2006:0631:FIN:EN:PDF

Comisión Europea. (2003). Hacia una asociación madura. Intereses comunes y desafíos en las relaciones UE-China (COM 533 final). http://eur-lex.europa.eu/ legalcontent/ES/TXT/PDF/?uri=CELEX:52003DC0533yfrom=ES

Comisión Europea y Alto Representante de la Unión para Asuntos Exteriores y Política de Seguridad. (2016). Comunicación conjunta al Parlamento Europeo y al Consejo: Componentes de una nueva estrategia de la UE respecto a China. http://eur-lex.europa.eu/legal-content/ES/TXT/?uri=LEGISSUM:r14206

Comisión Nacional para el Desarrollo y la Reforma, Ministerio de Asuntos Exteriores y Ministerio de Comercio de China. (2015). Vision and Actions on Jointly Building Silk Road Economic Belt and 21st-Century Maritime Silk Road. http://en.ndrc.gov.cn/newsrelease/201503/t20150330_669367.html

Consejo de Estado de China. (2018). China's Arctic Policy. http:/english.gov.cn/ archive/white_paper/2018/01/26/content_281476026660336.htm

Consejo Europeo. (2009). 11th EU-China Summit Joint Press Communiqué. http:// www.consilium.europa.eu/uedocs/cms_data/docs/pressdata/en/er/107965.pdf

Consejo Europeo. (9, detiembre, 2006). Ninth EU-China Summit Helsinki. Joint Statement 12642/06 (Presse 249). http://www.consilium.europa.eu/ueDocs/ cms_Data/docs/pressData/en/er/90951.pdf 
Dadush, U., Domínguez-Jiménez, M., y Gao, T. (2019). The State of China-European Union Economic Relations. Working Paper 9, Bruegel, 1-26. https:// www.bruegel.org/wp-content/uploads/2019/11/WP-2019-09-China-final.pdf

Díaz Barrado, C. (2016). La Unión Económica Euroasiática: Un paso crucial para la integración en el continente europeo y en el espacio euroasiático. Revista General de Derecho Europeo, 40. https:/www.iustel.com/v2/revistas/deta1 le_revista.asp?id_noticia $=417866 \& \mathrm{~d}=1$

Dirección General de Comercio de la Comisión Europea. (2018) European Union, Trade in Goods whit China. Bruselas.

Dokos, T. (2012). Who Lost Greece? The Geopolitical Consequences of the Greek Crisis. Policy Paper, 18, 1-23.

Esteban, M. y Li Y. (2017). Demystifying the Belt and Road Initiative: Scope, Actors and Repercussion for Europe. Duisburger Arbeitspapiere Ostasienwissenschaften 117/2017 (pp. 1-24). University of Duisburg-Essen, Institute of East Asian Studies.

Eurostat. (2020) China-European Union Trade 2012-2018. Bruselas (actualizado al 16 de julio).

Fallon, T. (8 de noviembre, 2019). Germany's Faustian Bargain with China. The Diplomat, https://thediplomat.com/2019/11/ germanys-faustian-bargain-with-china/

Feng, Z. (2008). A Chinese Perspective on China-European Relations. En G. Grevi y A. de Vasconcelos (Eds.), Partnerships for Effective Multilateralism, Chaillot Paper 109. European Union Institute for Security Studies.

Ghiasy, R. y Zhou J. (2017). The Silk Road Economic Belt: Considering Security Implications and EU-China Cooperation Prospects. SIPRI-Friedrich Ebert Stiftung, i-60.

Governo della Repubblica Italiana. (12 de marzo, 2019). Via della Seta, il testo dell'intesa tra l'Italia e la Cina: La versione inglese e la traduzione in italiano. Corriere della Sera. Roma.

Gonçalves, A. (2017). China's 'One Belt, One Road' Initiative. Just Economics?. Pyrex Journal of Political Science And International Relations, 3(2), 13-29.

Holslag, J. (2017). How China's New Silk Road Threatens European Trade. The International Spectator, 52(1), 46-60.

Hong, Z. (2017). An Overview of the China-EU Strategic Partnership (2003-2013). En Z. Hong (Ed.), China-EU Relations: Reassessing the China-EU Comprehensive Strategic Partnership (pp. 3-31). Social Sciences Academic Press y Springer. 
Huliaras, A. y Petropoulos S. (2014). Shipowners, Ports and Diplomats: the Political Economy of Greece's Relations with China. Asia Europe Journal, 12(3), 215-230.

Jones, D. A. y Liu, H. (2017). Management of Chinese Foreign Direct Investment: "One Belt, One Road" Across Eurasia to Africa and Europe Amidst Maritime Tensions in the South China Sea Region. International Relations and Diplomacy, 5(8), 486-500.

Juncker, J-C. (2015). The EU and China: A Solid Partnership - Speech by President Juncker at the EU-China Business Summit. http:/europa.eu/rapid/ press-release_SPEECH-15-5278_en.htm.

Kaplan, Y. (2017). China's OBOR as a Geo-Functional Institutionalist Project. Baltic Journal of European Studies, 7(1), 7-23.

Kavalski, E. (2013), The Struggle for Recognition of Normative Powers: Normative power Europe and normative power China in Context. Cooperation and Conflict, 48(2), 247-267.

Keohane, R. y Nye, J. (2005). La interdependencia en la política mundial. En A. Borja (Ed.), Ensayos escogidos de Robert O. Keohane (pp. 91-124). Centro de Información y Docencia Económica.

Keohane, R. y Nye, J. (1988). La interdependencia en la política mundial. En R.O. Keohane y J. S. Nye (Ed.), Poder e interdependencia: La politica mundial en transición (pp. 15-38). Grupo Editor Latinoamericano.

Le Corre, P. Pollack, J. (2017). China's rise: what about a Transatlantic Dialog? Asia-Europe Journal, 15(2), 147-160.

Liu, X. (26, enero, 2018). China proposes 'Polar Silk Road' cooperation in Arctic region. Global Times. http://www.globaltimes.cn/content/1086740.shtml

Li, M. (2016). China-EU Relations: Rivalry Impedes Strategic Partnership. En J. Wang y W. Song (Eds.), China, the European Union, and the International Politics of Global Governance (pp.13-28). Palgrave-Macmillan.

Malmström, C. (2015). Modernising Trade Policy - Effectiveness and Responsibility. http://trade.ec.europa.eu/doclib/docs/2015/july/tradoc_153621.pdf.

Manners, I. (2009). The Concept of Normative Power in World Politics. DIIS Brief. Danish Institute for International Studies, 1-5.

Parlamento Europeo. (Abril, 2019). Towards a New EU Policy approach to China, 21st EU-China Summit. -https://www.europarl.europa.eu/RegData/etudes/ ATAG/2019/637913/EPRS_ATA(2019)637913_EN.pdf

Pomfret, R. (2018). The Eurasian Land Bridge. The Role of Service Providers in Linking the Regional Value Chains in East Asia and the European Union. 
ERIA Discussion Paper Series 2018-01, 1-19). Economic Research Institute for ASEAN and East Asia.

Psaropoulos, J. (2019). Greece and China hail Strategic Partnership, as US and EU look on. Al Jazeera News, Atenas, 11 de noviembre. https://www. aljazeera.com/news/2019/11/greece-china-hail-strategic-partnershipeu-191111170150762.html

Rémond, M. (2007). The EU's Refusal to grant China "Market Economy Status" (MES). Asia Europe Journal, 5(3), 345-356.

Reuter, E. (2007). China-EU: A New Agenda. Asia Europe Journal, 5(2), 171-179.

Richet, X. (2018). The 16+1 Format: Chinese presence in Fragmented Markets on the Periphery of Europe. Working Paper, 21, 1-16. China-CEE Institute.

Richet, X., Ruet J., y Wang X. (2017). New Belts and Roads: Redrawing EU-China Relations. En A. Amighini (Ed.), China's Belt and Road: a Game Changer (pp. 97-119). Edizioni Epoké - ISPI.

Reuters. (2016). Piraeus Port shareholders approve COSCO Concession Deal. Reuters, 10 de junio. http://www.reuters.com/article/ us-eurozone-greece-privatisation-protest-idUSKCNOYW0ON

Rocha Pino, M. J. (2018). La asociación estratégica China-Unión Europea durante el periodo 2010-2018: Interdependencia económica y tensiones normativas. Revista Electrónica de Estudios Internacionales, 36, 1-28. http://www.reei. org/index.php/revista/num36

Scott, D. (2009). From Brussels to Beijing: Comparing the Regionalization Strategies of the EU and China. En E. Kavalski (Ed.), China and the Global Politics of Regionalization (pp. 109-119). Ashgate.

Servicio Europeo de Acción Exterior. (2016). Una visión común, una actuación conjunta: Una Europa más fuerte. Estrategia global para la política exterior y de seguridad de la Unión Europea. https:/europa.eu/globalstrategy/sites/ globalstrategy/files/eugs_es_version.pdf.

Servicio Europeo de Acción Exterior y Ministerio de Asuntos Exteriores de China. (2013). EU-China 2020 Strategic Agenda for Cooperation. http://eeas.europa.eu/china/docs/20131123_agenda_2020_en.pdf.

Statistisches Bundesamt. (2018). Ranking of Germany's Trading Partners in Foreign Trade. https://www.destatis.de/EN/FactsFigures/NationalEconomyEnvironment/ForeignTrade/Tables/OrderRankGermanyTradingPartners. pdf?__blob=publicationFile 
Steinbuka, I., Muravska T., y Kuznieks A. (2017). Cooperation Formats of China and Europe: Synergies and Divergences. Baltic Journal of European Studies, 7(1), 97-117.

Xi, J. (2017). Xi Jinping's keynote speech at the World Economic Forum. http:// www.china.org.cn/node_7247529/content_40569136.htm

Xi, J. (2013). President Xi Jinping delivers Important Speech and Proposes to build a Silk Road Economic Belt with Central Asian Countries. http://www.fmprc. gov.cn/mfa_eng/topics_665678/xjpfwzysiesgjtfhshzzfh_665686/t1076334. shtml. 